\title{
The Primordial 3-Helium Abundance At Last?
}

\author{
T. M. Bania \\ Institute for Astrophysical Research, Boston University, Boston, MA
}

Robert T. Rood

Department of Astronomy, University of Virginia, Charlottesville, VA

Dana S. Balser

National Radio Astronomy Observatory, Green Bank, WV

\begin{abstract}
We summarize the past 17 years of our efforts to determine the cosmic abundance of the ${ }^{3} \mathrm{He}$ isotope. The vast majority of our ${ }^{3} \mathrm{He}^{+}$ observations were made with the NRAO 140 Foot telescope in Green Bank, WV. The 140 Foot ceased operations in July 1999 so that NRAO could prepare to commission its replacement, the Green Bank Telescope (GBT). Our ${ }^{3} \mathrm{He}$ experiment was the last scientific program at the 140 Foot. It is thus poignant and timely for us to reassess the astrophysical context of our ${ }^{3} \mathrm{He}$ results. Here we argue that the existence of "The ${ }^{3} \mathrm{He}$ Plateau" for our sample of simple sources and recent advances in the understanding of the evolution of solar analog stars together suggest that we can finally estimate the primordial abundance of ${ }^{3} \mathrm{He}$. Our current best estimate for the primordial abundance is ${ }^{3} \mathrm{He} / \mathrm{H}=(1.5 \pm 0.6) \times 10^{-5}$ (s.e.) by number.
\end{abstract}

\section{Introduction}

3-Helium is one of the isotopes produced in the Big Bang. Standard models for stellar evolution predict that it is also produced, perhaps in prodigious amounts, by Solar-type stars. Thus measurements of the ${ }^{3} \mathrm{He}$ abundance are of interest both for cosmology and the chemical evolution of the Galaxy. For almost two decades we have been determining the ${ }^{3} \mathrm{He}$ abundance using measurements of the $3.5 \mathrm{~cm}$ hyperfine transition of ${ }^{3} \mathrm{He}^{+}$. Our 66 source observational sample includes evolved stars (planetary nebulae) and star forming (H II) regions which are located throughout the Milky Way's gaseous disk, from the Galactic Center to the far reaches of the outer Galaxy. Although the observations are technically quite challenging, we have detected ${ }^{3} \mathrm{He}$ over a larger fraction of the Galaxy than any other light isotope. We are finding that Milky Way $\mathrm{H}$ II regions are not ${ }^{3} \mathrm{He}$ enriched and, furthermore, that the distribution of ${ }^{3} \mathrm{He}$ in the Galaxy cannot be easily explained by chemical evolution models. One thing is clear: real stars haven't heard about standard stellar models-there is no evidence that Solar-type stars are enriching the Galaxy in ${ }^{3} \mathrm{He}$. On the other hand we 


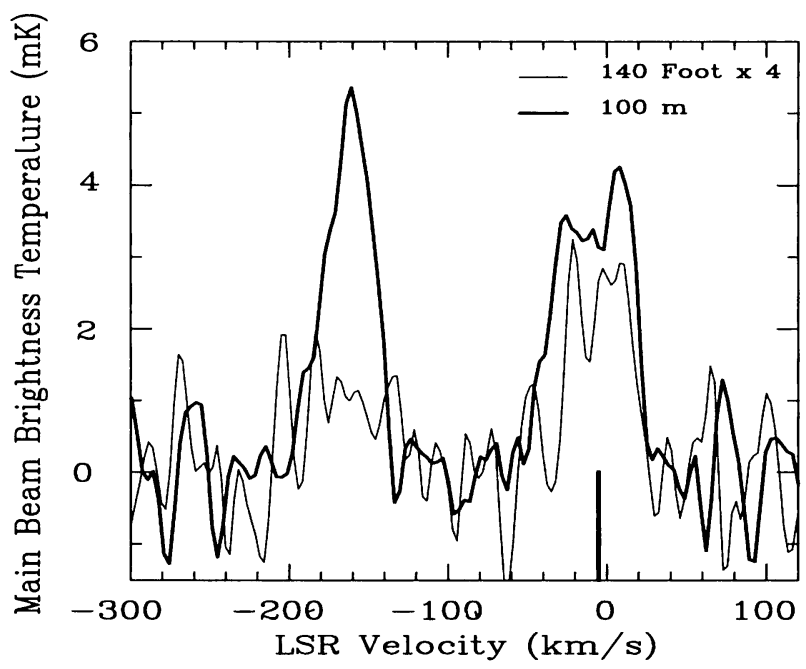

Figure 1. Confirmation of the detection of ${ }^{3} \mathrm{He}^{+}$emission from the planetary nebula NGC 3242 . The thick curve is the spectrum taken with the MPIfR 100 meter telescope. The thin curve is the spectrum taken with the NRAO 140 foot telescope with the observed intensity multiplied by a factor of 4 . The vertical line at $-5.3 \mathrm{kms}^{-1}$ flags the ${ }^{3} \mathrm{He}^{+}$emission at the LSR velocity of NGC 3242 . The prominent feature in the MPIfR spectrum is the H171 $\eta$ recombination line.

find lots of ${ }^{3} \mathrm{He}$ in some planetary nebulae-the ejecta of Solar-type stars. This seemingly contradictory situation has led to "The ${ }^{3} \mathrm{He}$ Problem" (Galli et al. 1997). Here we review the problem as a whole and reflect upon how cosmological interpretations of light element abundances are made so very much more difficult when one is burdened by a surfeit of sources.

\section{The 3-Helium Experiment}

We derive the ${ }^{3} \mathrm{He}$ abundance in Milky Way sources using measurements of the hyperfine transition of ${ }^{3} \mathrm{He}^{+}$at $3.46 \mathrm{~cm}$ wavelength together with numerical models for the density and ionization structure of each source (see, e.g., Balser et al. 1997 [BBRW97], 1999 [BBRW99]; Bania et al. [BBRWW97]). Because the Milky Way interstellar medium (ISM) is optically thin at centimeter wavelengths, our source sample probes a larger volume of the Galactic disk than does any other light element tracer of Galactic chemical evolution. The source sample is currently comprised of 6 planetary nebulae $(\mathrm{PNe})$ and $60 \mathrm{H}$ II regions. For technical reasons the bulk of the PNe observations were made using the $100 \mathrm{~m}$ telescope of the MPIfR in Effelsberg, Germany, whereas the majority of the $\mathrm{H}$ II observations were made using the 140 Foot telescope of the NRAO in Green Bank, WV, USA. 


\subsection{Planetary Nebulae}

Standard models for the evolution of solar analog stars predict (1) that these objects should produce copious amounts of ${ }^{3} \mathrm{He}$ during their main sequence lifetimes, (2) that the ${ }^{3} \mathrm{He}$ is mixed to the surface during the first dredge-up low on the red giant branch, and (3) that ${ }^{3} \mathrm{He}$ rich material flows into the ISM in stellar winds along the first and second red giant branches and in the final planetary nebula. The subsequent ${ }^{3} \mathrm{He}$ enrichment of the ISM should be significant. If we are to understand the chemical evolution of the Galaxy, it is important to pin down definitively the contribution of PNe to the ${ }^{3} \mathrm{He}$ abundance. Galli et al. (1997) show how the $\mathrm{PNe}{ }^{3} \mathrm{He}$ abundances and estimates of the PN progenitor masses together provide a direct test of stellar evolution theory.

It is technically quite challenging to detect ${ }^{3} \mathrm{He}^{+}$emission from $\mathrm{PNe}$ even though the theoretically expected abundances, ${ }^{3} \mathrm{He} / \mathrm{H} \sim 1-10 \times 10^{-4}$, are a factor of ten or more larger than that measured for Galactic $\mathrm{H}$ iI regions. Unfortunately typical PNe angular sizes of $\sim 20^{\prime \prime}$ are much smaller than the beamsizes of even the largest centimeter wave telescopes, so the ${ }^{3} \mathrm{He}^{+}$emission from these objects is weakened by geometrical dilution. For the MPIfR $100 \mathrm{~m}$ telescope the typical dilution factor is $\sim 12$ and for the NRAO 140 Foot it is $\sim 92$.

Because of this geometrical dilution we used the MPIfR $100 \mathrm{~m}$ telescope to search for ${ }^{3} \mathrm{He}^{+}$emission from PNe (Rood, Bania, \& Wilson 1992; BBRW97). Even so, we were working at the sensitivity limit of the $100 \mathrm{~m}$ telescope. Our survey sample of six PNe was therefore chosen to maximize the likelihood of ${ }^{3} \mathrm{He}^{+}$ detections, and so it was highly biased. For example, we chose sources that were $\sim 500 \mathrm{pc}$ from the Galactic plane. This criterion increased the likelihood that a given PN was part of the old/thick disk or halo population. On the average, such $\mathrm{PNe}$ would have low progenitor masses, longer main-sequence lifetimes, and, thus, presumably higher ${ }^{3} \mathrm{He}$ abundances. Furthermore, our PNe sample showed no evidence in the nebular gas of nuclear processing on the RGB or AGB. This excluded objects where the gas had undergone CNO processing since ${ }^{3} \mathrm{He}$ would have also been depleted in such objects. Specifically, our PNe sample had low $\mathrm{He}, \mathrm{N}$, and ${ }^{13} \mathrm{C}$ abundances.

This PNe survey detected ${ }^{3} \mathrm{He}$ emission in NGC 3242 and probably also detected it in NGC 6543 and NGC 7009. These detections showed that there is some stellar production of ${ }^{3} \mathrm{He}$. The abundances derived for the detected sources are consistent with the idea that ${ }^{3} \mathrm{He}$ is produced in significant quantities by at least some stars with masses in the range $1-2 M_{\odot}$. Finally, in BBRW97 we argued that the ${ }^{3} \mathrm{He}^{+}$emission was just below the detection threshold for many of the sources.

Because we were working at the sensitivity limit of the $100 \mathrm{~m}$ telescope it was important that we confirm these results. Since the NRAO 140 Foot telescope had an additional factor of ten geometrical dilution above that of the $100 \mathrm{~m}$, we concentrated our efforts on the PN NGC 3242. Figure 1 shows that after a 270 hour integration we have confirmed the presence of ${ }^{3} \mathrm{He}^{+}$emission from the PN NGC 3242 (Balser, Rood, \& Bania 1999). Both spectrometers show that the observed ${ }^{3} \mathrm{He}^{+}$hyperfine emission has a double-peaked line profile structure. This line shape naturally arises if NGC 3242 is modeled as a dense core surrounded by a very low density expanding shell. After modeling this source we conclude that a ${ }^{3} \mathrm{He} / \mathrm{H}$ abundance ratio by number of $(2-5) \times 10^{-4}$ is 


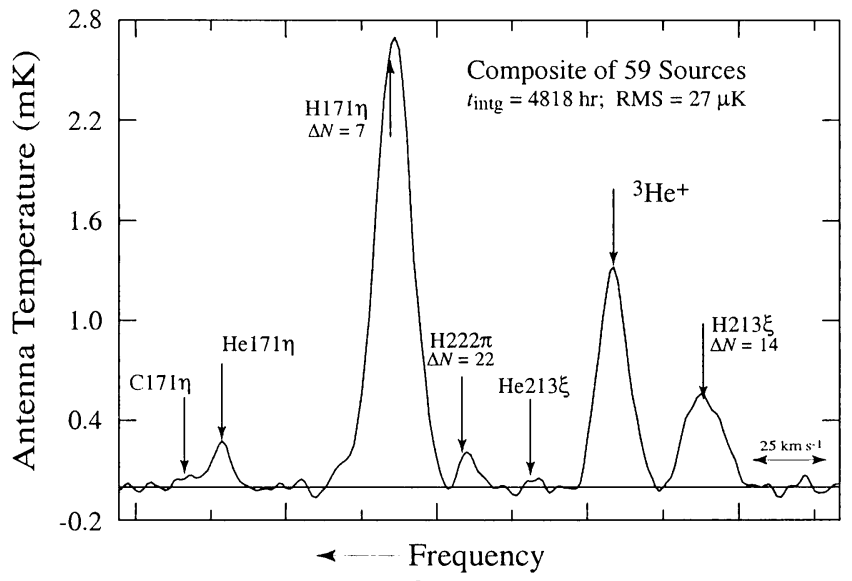

Figure 2. The grand average of ${ }^{3} \mathrm{He}$ observations obtained with the Green Bank 140 Foot Telescope from 1982 through 1999. The spectrum is a composite of 59 Galactic $\mathrm{H}$ II regions. The observed RMS noise after almost 5000 hours of integration is just as predicted by the radiometer equation. This shows that we are correctly dealing with possible sources of systematic error like spectral baseline removal.

consistent with both observed spectra. This result confirms stellar production of ${ }^{3} \mathrm{He}$ by some yet to be determined fraction of the Galactic PN population.

\section{2. $\quad$ H II Regions}

Because $\mathrm{H}$ II regions are zero-age objects when compared to the age of the Galaxy, the elemental abundances of these nebulae chronicle the results of billions of years Galactic chemical evolution. Measurement of the present ${ }^{3} \mathrm{He}$ abundance should therefore be an important diagnostic of chemical evolution in the Galaxy. Standard stellar ${ }^{3} \mathrm{He}$ nucleosynthesis predicts that the ${ }^{3} \mathrm{He} / \mathrm{H}$ abundance ratio should grow with time and be higher in those parts of the Galaxy where there has been substantial stellar processing. Specifically: (1) the protosolar value should be less than that found in the present ISM; and (2) there should be $\mathrm{a}^{3} \mathrm{He} / \mathrm{H}$ abundance gradient across the Galactic disk with the highest abundances occurring in the highly-processed inner Galaxy.

We have now accumulated a sample of $60 \mathrm{H}$ II regions that sample the entire Galactic disk, from the Galactic Center to the far reaches of the outer Galaxy. During the past few years at Green Bank we have dramatically increased the number of $\mathrm{H}$ II regions with ${ }^{3} \mathrm{He}$ detections due to a combination of technical improvements and better astrophysical insight in our selection of candidate sources. We have benefited from the new generation of high sensitivity receivers constructed by the NRAO for the GBT. We have also learned something about source selection: in the past few years nearly all of our candidate $\mathrm{H}$ II regions have yielded ${ }^{3} \mathrm{He}$ detections.

The selection of ${ }^{3} \mathrm{He}$ targets is counter-intuitive because the ${ }^{3} \mathrm{He}^{+}$hyperfine line strength is proportional to the source density, while one usually thinks of $\mathrm{H}$ II regions in terms of radio continuum or recombination line strength both of 


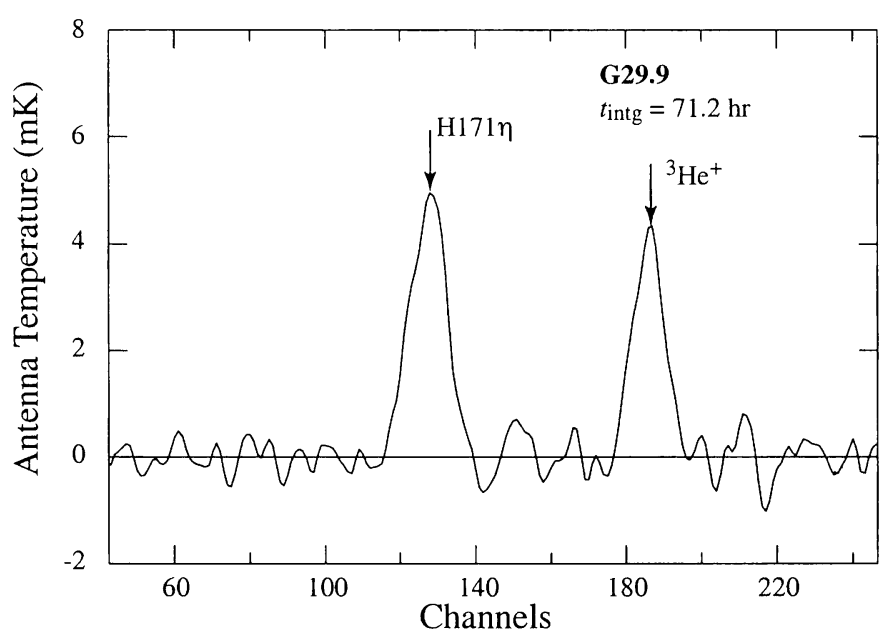

Figure 3. $\quad{ }^{3} \mathrm{He}^{+}$spectrum of the $\mathrm{H}$ II region G29.9. The spectrum has a velocity resolution of $8.1 \mathrm{~km} \mathrm{~s}^{-1}$. Vertical arrows flag the expected positions of the detected lines. Although relatively obscure as $\mathrm{HII}$ regions go, G29.9 has one of the strongest ${ }^{3} \mathrm{He}^{+}$lines we have observed.

which depend on the square of the density. The ${ }^{3} \mathrm{He}^{+}$line strength depends on the ${ }^{3} \mathrm{He}^{+}$abundance ratio and a number of other factors:

$$
T_{L}^{A}\left({ }^{3} \mathrm{He}^{+}\right) \propto \frac{N\left({ }^{3} \mathrm{He}^{+}\right)}{N\left(\mathrm{H}^{+}\right)} \frac{\left(T_{C}^{A} D\right)^{1 / 2} T_{e}^{1 / 4}\left(\theta_{\mathrm{obs}}^{2}-\theta_{\mathrm{beam}}^{2}\right)^{3 / 4}}{\Delta v\left({ }^{3} \mathrm{He}^{+}\right)\left[\ln \left(5.717 \times 10^{-3} T_{e}^{3 / 2}\right)\right]^{1 / 2} \theta_{\mathrm{obs}}}
$$

where $T_{\mathrm{L}}^{\mathrm{A}}$ and $\Delta v$ are the antenna temperature and FWHM of the ${ }^{3} \mathrm{He}^{+}$line, $D$ is the nebular distance, $T_{\mathrm{C}}^{\mathrm{A}}$ and $\theta_{\mathrm{obs}}$ are the antenna temperature and observed FWHM angular size of the continuum emission, $\theta_{\text {beam }}$ is the telescope's FWHM beam, and $T_{e}$ is the nebular electron temperature. For $\mathrm{H}$ II regions much larger than the telescope beam we can select targets using the criterion: $T_{L}^{A}\left({ }^{3} \mathrm{He}^{+}\right) \sim$ $\sqrt{T_{C}^{A} D \theta_{\mathrm{obs}}}$. This is the case since we can neglect the weak dependence on $T_{e}$ and because we do not know either ${ }^{3} \mathrm{He}^{+} / \mathrm{H}^{+}$or $\Delta v$. Thus big, distant $\mathrm{H}$ il regions could be potential ${ }^{3} \mathrm{He}^{+}$targets even if their continuum emission is weak.

Armed with this knowledge we included H II regions like S209 in our early observing list along with more famous sources like W43. Still we did not have the temerity to push this reasoning to the limit. We have now found, however, that this selection criterion is valid for even the wimpiest known $\mathrm{H}$ II regions. It is this realization that has led to the dramatic increase in the number of ${ }^{3} \mathrm{He}^{+}$ sources discovered at Green Bank during the last several years. As expected, these are all large, low density, diffuse $\mathrm{H}$ II regions.

\subsection{The 3-Helium Plateau}

We have found source-to-source variations in the ${ }^{3} \mathrm{He} / \mathrm{H}$ abundance of at least a factor of five for Galactic Hirregions (e.g. BBRW99). The observed abundance 


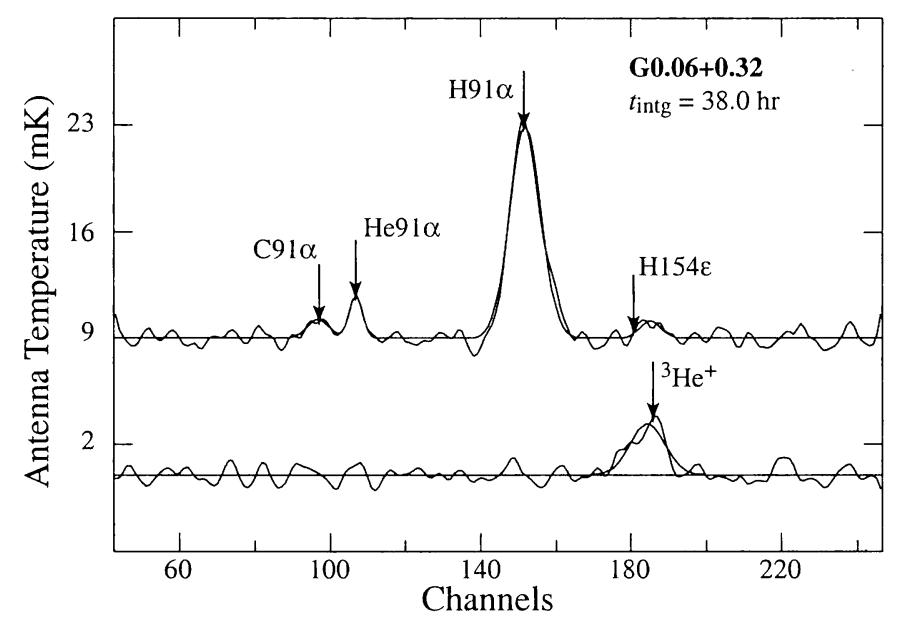

Figure 4. Spectrum of the $\mathrm{H}$ II region G0.06+0.32. This is an example of the large, diffuse sources detected during the past few years with the NRAO 140 Foot telescope. The top figure is the H91 $\alpha$ spectrum; the bottom figure is the ${ }^{3} \mathrm{He}^{+}$spectrum. The expected centers of detected transitions are flagged. The $\mathrm{H} 91 \alpha$ line is only 4 times stronger than the ${ }^{3} \mathrm{He}^{+}$line; in tradional $\mathrm{H}$ II regions like W43 the $\mathrm{H} 91 \alpha$ line is several hundred times stronger than the ${ }^{3} \mathrm{He}^{+}$line. Even though G0.06+0.32 is roughly in the direction of the Galactic center, it is actually $11.5 \mathrm{kpc}$ on the other side of the center.

pattern, however, cannot be easily explained by existing chemical evolution models. There is no evidence for a gradient in the Galaxy. Nor do the H II regions show any evidence for systematic stellar ${ }^{3} \mathrm{He}$ enrichment during the last $4.5 \mathrm{Gyr}$. Indeed, we have made little progress in understanding how the few $\mathrm{H}$ II regions with high ${ }^{3} \mathrm{He}$ got that way.

Our goal is to derive the most accurate possible ${ }^{3} \mathrm{He} / \mathrm{H}$ abundances, developing in the process a theoretical framework that will allow us to understand quantitatively the inherent systematic errors. As is the case for any cosmic abundance determination, converting the measured ${ }^{3} \mathrm{He}^{+}$column density into an abundance ratio relative to hydrogen, ${ }^{3} \mathrm{He} / \mathrm{H}$, is nontrivial. For the case of ${ }^{3} \mathrm{He}$ this conversion depends on the density and ionization structure of each nebula. In BBRW99 we developed numerical models for our ${ }^{3} \mathrm{He}^{+}$sources which account for density structure. We discovered that a major advantage of diffuse $\mathrm{H}$ II regions is that they tend to be "simple," i.e., the H II region has a relatively simple density structure. This allows us to derive abundances from the observed ${ }^{3} \mathrm{He}^{+}$line parameters without recourse to the rather complex modeling required for most classic $\mathrm{H}$ II regions (see BBRW99). Because the abundance corrections for simple sources are negligible, these sources can in principle yield the most accurate ${ }^{3} \mathrm{He} / \mathrm{H}$ abundance ratios attainable.

At present our H II region sample has 19 sources that are "simple" in the sense just described. As was the case for the full $\mathrm{H}$ II region sample, our sample of simple sources shows no evidence for an abundance gradient in the Galaxy, no evidence for any stellar ${ }^{3} \mathrm{He}$ enrichment during the last $4.5 \mathrm{Gyr}$, and no trend 


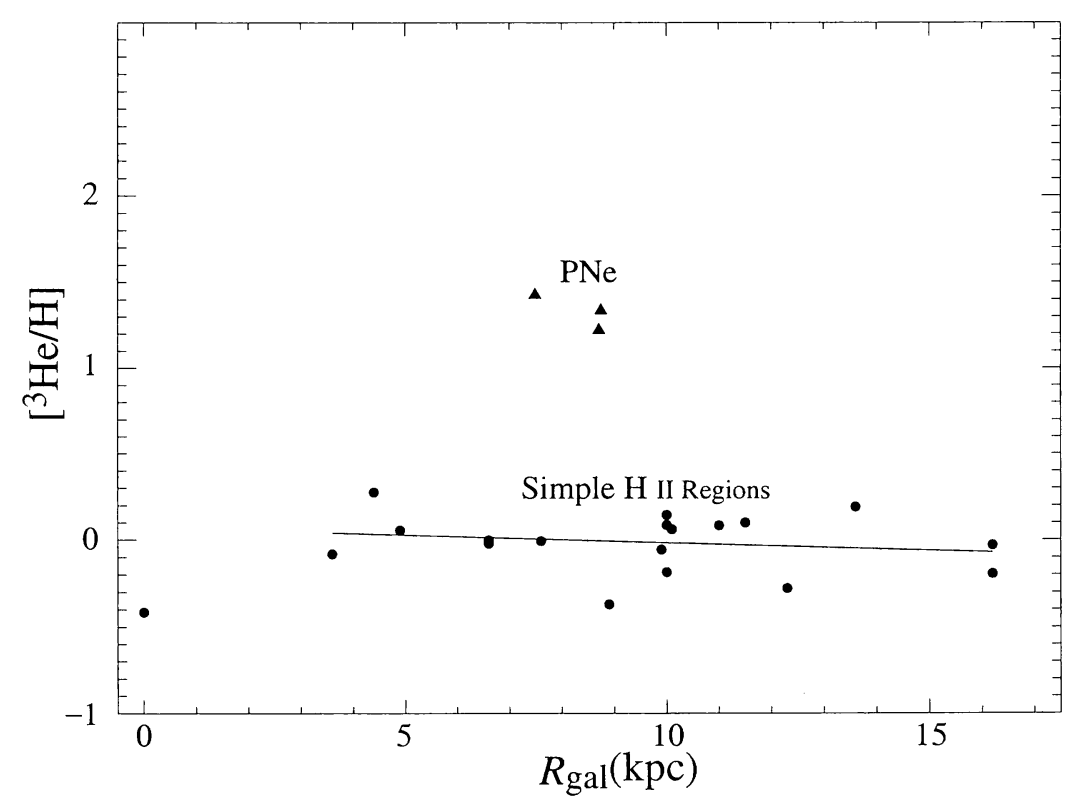

Figure 5. $\quad\left[{ }^{3} \mathrm{He} / \mathrm{H}\right]\left(=\log \left({ }^{3} \mathrm{He} / \mathrm{H}\right)-\log \left({ }^{3} \mathrm{He} / \mathrm{H}\right)_{\odot}\right)$ abundances plotted as a function of galactocentric radius for the sample of "simple" Galactic $\mathrm{H}$ II regions (see text). Also shown are the abundances derived for the planetary nebulae NGC 3242, NGC 6543, and NGC 7009. The best linear fit to the data is shown. The solar abundance $\left({ }^{3} \mathrm{He} / \mathrm{H}\right)_{\odot}$ was taken to be $1.5 \times 10^{-5}$. The point near $R_{\text {gal }}=0$ is the H II region G1.1. It is not included in the averages or fits because the Galactic center region is thought to have a different chemical enrichment history than the disk.

of abundance with metallicity. (We can derive an $[\mathrm{O} / \mathrm{H}]$ abundance from our nebular models using the relationship between nebular electron temperature and [O/H] derived by Shaver et al. 1983.)

But this result is a trend: simple ${ }^{3}$ He sources show absolutely no abundance gradient with respect to either galactocentric radius or metallicity. The dispersion of ${ }^{3} \mathrm{He}$ abundances, moreover, is very small-comparable to that of the famous "Lithium Plateau". The "3 He Plateau" defined by our sample of simple sources has an average ${ }^{3} \mathrm{He}^{+} / \mathrm{H}^{+}$abundance ratio by number of $(1.37 \pm 0.54) \times 10^{-5}$ (s.e.) by number.

Here we make a preliminary ionization correction to these abundances using the observed source ${ }^{4} \mathrm{He}^{+} / \mathrm{H}^{+}$abundance ratio, $y_{4}$, measured via the $\mathrm{H}$ and $\mathrm{He}$ $91 \alpha$ recombination transitions. Since we have never found ${ }^{4} \mathrm{He}^{++}$emission in any of our $\mathrm{H}$ II regions, we assume that $y_{4}$ is a reasonably accurate estimate of a source's helium ionization state. To correct for source ionization we demand that $y_{4}=0.10$. That is, for sources with $y_{4}<0.10$, we scale the ${ }^{3} \mathrm{He}^{+} / \mathrm{H}^{+}$abundance by the ratio $0.10 / y_{4}$, otherwise we set ${ }^{3} \mathrm{He} / \mathrm{H}={ }^{3} \mathrm{He}^{+} / \mathrm{H}^{+}$. Nine of the simple sources need to be ionization corrected. The "3 He Plateau" defined by our 
ionization corrected sample of simple sources has an average ${ }^{3} \mathrm{He} / \mathrm{H}$ abundance ratio of $(1.50 \pm 0.58) \times 10^{-5}$ (s.e.) by number. This value and the absence of a gradient are completely consistent with the proto-solar system and local ISM values of ${ }^{3} \mathrm{He}$ (Gloeckner 2000).

\section{Astrophysical Implications: "The 3-Helium Problem"}

We have shown that at least some low-mass stars have produced significant amounts of ${ }^{3} \mathrm{He}^{+}$. Yet our observations of ${ }^{3} \mathrm{He}$ in zero-age $\mathrm{H}$ II regions imply that essentially no stellar enrichment has occurred over the last 4.5 Gyr (BBBRW99). Chemical evolution models of the Galaxy indicate that to account for the observed abundances in $\mathrm{H}$ II regions, no more than 15 or $20 \%$ of low-mass stars can produce ${ }^{3} \mathrm{He}$ like the progenitor of NGC 3242 did (Galli et al. 1997).

\subsection{Stellar Production of 3-Helium}

As early as 1984 (Rood, Bania, \& Wilson 1984, §V.c) we suggested that the low abundances of ${ }^{3} \mathrm{He}$ which we were just then finding indicated a breakdown in standard stellar models. We also pointed out that there might be some connection with observed $\mathrm{Li}$ abundances and $\mathrm{CNO}$ anomalies. In the intervening years the case for low abundances became stronger and chemical evolution models became far more sophisticated. Chemical evolution models that adopted standard stellar ${ }^{3} \mathrm{He}$ nucleosynthesis overproduced ${ }^{3} \mathrm{He}$. All Galactic evolution models which match the other observational constraints (e.g., star formation rate, gas and total mass density, mass infall rate, IMF, etc.) predict ${ }^{3} \mathrm{He}$ abundances that are inconsistent with those observed both locally and globally in the Milky Way unless they adopt alternative nucleosyntheses with a strongly reduced ${ }^{3} \mathrm{He}$ contribution from low- and intermediate-mass stars (Tosi 2000).

In the past few years some stellar models with non-standard assumptions have been shown to be ${ }^{3} \mathrm{He}$ non-producers. In particular, Charbonnel, et al. $(1995,1998,1998 \mathrm{a}, 1998 \mathrm{~b})$ investigate rotationally driven mixing processes in an effort to explain various observed abundance anomalies. They show that while observed ${ }^{12} \mathrm{C} /{ }^{13} \mathrm{C}$ ratios agree well with standard models for stars less luminous than the red giant luminosity function bump, the ratios above the bump drop indicating non-canonical mixing. They attribute this continued mixing to rotationally driven turbulence. Their models nicely account for a number of observables like the ${ }^{12} \mathrm{C} /{ }^{13} \mathrm{C}$ ratios in red giants in open clusters and with Hipparchos distances. Stars with low ${ }^{12} \mathrm{C} /{ }^{13} \mathrm{C}$ ratios would have a correspondingly low ${ }^{3} \mathrm{He}$. A few stars, presumably slow rotators, preserve a high value for ${ }^{12} \mathrm{C} /{ }^{13} \mathrm{C}$. In these the ${ }^{3} \mathrm{He}$ would also survive allowing for high ${ }^{3} \mathrm{He}$ in a few PNe. The fraction of stars with high ${ }^{12} \mathrm{C} /{ }^{13} \mathrm{C}$ is approximately that allowed by the ${ }^{3} \mathrm{He}$ constraint on chemical evolution models. The Charbonnel models illustrate a subtle but important point: the stars with low ${ }^{12} \mathrm{C} /{ }^{13} \mathrm{C}$ have indeed destroyed most of the ${ }^{3} \mathrm{He}$ produced on the main sequence. Still the resulting ${ }^{3} \mathrm{He}$ is comparable to or higher than the initial value. As far a chemical evolution goes such stars are non-producers rather than destroyers of ${ }^{3} \mathrm{He}$.

Sackmann \& Boothroyd (1999 and references therein) have also explored non-standard mixing processes. Their parametric approach is not as tightly tied to a physical mechanism as is that of Charbonnel et al. It does not appear 
to be as tightly constrained by observations. They do, however, find a part of parameter space in which stars do indeed destroy ${ }^{3} \mathrm{He}$. If operative in all stars this mechanism could lead to net destruction of ${ }^{3} \mathrm{He}$. Additional observations of ${ }^{12} \mathrm{C} /{ }^{13} \mathrm{C}$ and other elements are crucial to delineate the character and statistics of these non-standard mixing processes.

\subsection{The Primordial 3-Helium Abundance}

The existence of the ${ }^{3} \mathrm{He}$ Plateau suggests that there is a floor to the ${ }^{3} \mathrm{He} / \mathrm{H}$ abundance. If, as seems plausible, stellar processing enriches the ISM ${ }^{3} \mathrm{He}$ abundance by a positive-definite (but perhaps nearly zero) amount, this floor value provides an upper limit to the primordial ${ }^{3} \mathrm{He}$ abundance produced by Big Bang Nucleosynthesis. That is, BBNS must not overproduce ${ }^{3} \mathrm{He}$ beyond the floor abundance of ${ }^{3} \mathrm{He} / \mathrm{H}=(1.5 \pm 0.6) \times 10^{-5}$ (s.e.) by number.

\section{Summary and Future Prospects}

During the past 20 years much progress has been made in understanding the Galactic abundance of ${ }^{3} \mathrm{He}$ and its temporal evolution. Although there are still outstanding issues regarding "The ${ }^{3} \mathrm{He}$ Problem," recent progress in nonstandard stellar mixing has provided new insight into the chemical evolution of ${ }^{3} \mathrm{He}$. When combined with the existence of "The ${ }^{3} \mathrm{He}$ Plateau" for our sample of "simple" Galactic H II regions, we now believe that we can estimate the primordial ${ }^{3} \mathrm{He}$ abundance.

There are still many more $\mathrm{H}$ II regions that can be studied. We were still detecting new ${ }^{3} \mathrm{He}$ sources with the NRAO 140 Foot telescope until the bitter end at 08:12 (EDT) 19 July, 1999. Indeed,we have already measured the ${ }^{3} \mathrm{He}^{+}$ line in many $\mathrm{H}$ II regions not included in Figure 5. It is the lack of adequate continuum measurements and even simple source modeling that prevent us from giving abundances now. The GBT and the newly upgraded Arecibo telescope will greatly enhance the sensitivity of the ${ }^{3} \mathrm{He}$ experiment. We are especially excited by the potential of the GBT as its unblocked-aperture design is optimal for the ${ }^{3} \mathrm{He}$ experiment.

Ours in the only project in the world making a systematic study of ${ }^{3} \mathrm{He}$ throughout the Galaxy. While our project has already continued for many years, we can continue to improve our understanding of the origin and evolution of ${ }^{3} \mathrm{He}$ by: (1) observing a larger sample of $\mathrm{PNe}$ to reduce the observational bias in our current sample; (2) improving the accuracy of our current PNe sample; (3) observing new $\mathrm{H}$ II regions chosen to test specific questions which have risen from current results; (4) obtaining improved spectra for a few crucial $\mathrm{H}$ II regions; (5) refining the emission line models of detected ${ }^{3} \mathrm{He}^{+}$sources; (6) making further improvements to the ${ }^{3} \mathrm{He}$ abundance determinations by constructing more sophisticated source models; and (7) searching for ${ }^{3} \mathrm{He}^{+}$emission from extragalactic $\mathrm{H}$ II regions.

After spending so long on a project it is amusing to reflect on how it may have come out differently. If we were starting the project now and applying for telescope time on a highly oversubscribed telescope, like the GBT, we might be forced to submit the minimum proposal to prove our point. We likely would propose observations of W43 and W51 in the inner Galaxy and the remote 
outer Galaxy H II region S209. We would make easy detections and find a somewhat lower ${ }^{3} \mathrm{He} / \mathrm{H}$ ratio than expected; there would be a gradient in ${ }^{3} \mathrm{He}$ consistent with our naive expectations. We would never get any more telescope time, because the TAC would respond "Nothing to be learned; standard model confirmed." Unfortunately the early 1980's Green Bank TACs were generous. We observed too many $\mathrm{H}$ II regions in the wrong order, and the situation was very confusing from our first observing run. We have learned many things since 1982: (1) too much data can be confusing; (2) The Green Bank kitchen staff can be enticed to cook spaghetti carbonara (but the raw egg part causes panic); $(3+)$ no more space; deferred to a future paper.

Acknowledgments. We thank Tom Wilson for his contributions to the ${ }^{3} \mathrm{He}$ project over the years and for making this paper necessary. The research was supported by a NATO Travel Grant and the U.S. National Science Foundation (AST 97-31484).

\section{References}

Balser, D. S., Bania, T. M., Rood, R. T., \& Wilson, T. L. 1997, ApJ, 483, 320 (BBRW97)

Balser, D. S., Bania, T. M., Rood, R. T., \& Wilson, T. L. 1999, ApJ, 510, 759 (BBRW99)

Balser, D. S., Rood, R. T., \& Bania, T. M. 1999, ApJ, 522, L73

Bania, T. M., Balser, D. S., Rood, R. T., Wilson, T. L., \& Wilson, T. A. 1997, ApJS, 113, 353 (BBRWW97)

Charbonnel, C. 1995, ApJ, 453, L41

Charbonnel, C. 1998, Space Science Reviews, 84, 199

Charbonnel, C., Brown, J. A., Wallerstein, G. 1998a, A\&A, 332, 204

Charbonnel, C., \& do Nascimento Jr, J. D. 1998b, A\&A, 336, 915

Galli, D., Stanghellini, L., Tosi, M., Palla, F. 1997, ApJ, 477, 218

Gloeckler, G. 2000, in this volume

Rood, R. T., Bania, T. M., and Wilson, T. L. 1984, ApJ, 280, 629

Rood, R. T., Bania, T. M., \& Wilson, T. L. 1992, Nature, 355, 618

Rood, R. T., Bania, T. M., Balser, D. S., \& Wilson, T. L. 1998, Space Science Reviews, 84, 185

Rood, R. T., Steigman, G., \& Tinsley, B. M. 1976, ApJ, 207, L57

Sackmann, I.-J. \& Boothroyd, A. I. 1999, ApJ, 510, 217

Shaver, P. A., McGee, R. X., Newton, L. M., Danks, A. C., \& Pottasch, S. R. 1983, MNRAS, 204, 53

Tosi, M. 2000, in this volume 